\title{
Entre doutes et engagements : un arrêt sur image à partir de l'histoire des femmes ( $2^{\text {ème }}$ partie)
}

Groupe Histoire des femmes, Myriam Cottias, Cécile Dauphin, Arlette Farge, Nancy L. Green, Danielle Haase-Dubosc, Danièle Poublan et Yannick Ripa

\section{(2) OpenEdition \\ 12 Journals}

Édition électronique

URL : https://journals.openedition.org/clio/1474

DOI : $10.4000 /$ clio. 1474

ISSN : 1777-5299

Éditeur

Belin

Édition imprimée

Date de publication : 1 avril 2005

ISBN : 2-85816-781-8

ISSN : $1252-7017$

\section{Référence électronique}

Groupe Histoire des femmes, Myriam Cottias, Cécile Dauphin, Arlette Farge, Nancy L. Green, Danielle Haase-Dubosc, Danièle Poublan et Yannick Ripa, « Entre doutes et engagements : un arrêt sur image à partir de l'histoire des femmes (2ème partie) », Clio. Histoire, femmes et sociétés [En ligne], 21 | 2005, mis en ligne le 06 juin 2005, consulté le 23 avril 2022. URL : http://journals.openedition.org/clio/1474 ; DOI : https://doi.org/10.4000/clio.1474

Ce document a été généré automatiquement le 23 avril 2022

Tous droits réservés 


\title{
Entre doutes et engagements : un arrêt sur image à partir de l'histoire des femmes ( $2^{\text {ème }}$ partie)
}

\author{
Groupe Histoire des femmes, Myriam Cottias, Cécile Dauphin, Arlette \\ Farge, Nancy L. Green, Danielle Haase-Dubosc, Danièle Poublan et Yannick \\ Ripa
}

\section{NOTE DE L'ÉDITEUR}

Le Groupe d'histoire des femmes du Centre de Recherches Historiques (EHESS-CNRS) fonctionne depuis 1978. Il a publié différents travaux sur la femme seule, la violence, la séduction et un essai d'historiographie dans les Annales ESC, 1986. Il est actuellement composé de Myriam Cottias, chargée de recherche au CNRS (CRPLC - Université des Antilles et de la Guyane, CRH), Cécile Dauphin, ingénieure de recherche au CNRS (CRH), Arlette Farge, directrice de recherche au CNRS (CRH), Dominique Godineau, maitre de conférences à l'université Rennes II (CRHISCO), Nancy L. Green, directrice d'études à l'EHESS (CRH), Danielle Haase-Dubosc, directrice de Reid Hall, directrice d'études de Columbia University à Paris, Christiane Klapisch-Zuber, directrice d'études à l'EHESS (CRH), Danièle Poublan, ingénieure d'études à l'EHESS (CRH), Yannick Ripa, maître de conférence à l'université Paris VIII.

Les contributions de Myriam Cottias, Cécile Dauphin, Arlette Farge et Danièle Poublan sont parues dans le numéro précédent.

Cet article collectif, dont la première partie a été publiée dans le numéro précédent, se poursuit selon les modalités indiquées dans l'introduction. À partir de mots clés, ou de notions utilisées depuis les années 1980, il s'agit de proposer à la fois un arrêt sur images et une histoire critique du vocabulaire. Désir aussi de politique et d'appréhension plus fine du réel. Sur ces questions, les points de vue n'engagent que leurs auteures. L'objet de CLIO, Histoire, Femmes et Sociétés n'est pas, en effet, de 
prendre position comme revue dans les débats politiques d'aujourd'hui, mais de fournir un espace intellectuel et institutionnel à l'histoire des femmes et du genre.

\section{Du patriarcat au genre}

1 Interrogée sur ce que lui évoque le mot patriarcat, la mémoire féministe répond «l'ennemi principal»; réponse sans doute aujourd'hui plus mécanique que pensée car, malgré la réédition du fameux texte de Christine Delphy ${ }^{1}$ et le débat qui s'en suivit, le terme ne fait plus florès. Il semble aujourd'hui avoir été remisé dans le seul tiroir des instruments d'analyse des anthropologues - encore que Françoise Héritier préfère dans sa théorie de la valence différentielle pointer «l'importance idéelle des structures de parenté ", et questionner la " domination masculine $»^{2}$; il a pareillement déserté la rue, la majorité des écrits féministes et la plupart des publications en sciences humaines.

Deux images superposées rendent compte de cette évidente désaffection. Scandé sur tous les tons, des slogans aux chansons, le patriarcat est bien dans les manifestations du Mouvement de libération des femmes le responsable d'une "condition féminine » opprimée; de ce fait, l'adversaire est clairement désigné par les féministes qui l'introduisent sur la scène politique ${ }^{3}$ et l'accusent avec les mots crus de l'époque ${ }^{4} .8$ mars 2003 : «La Marche des femmes des quartiers pour l'égalité et contre le ghetto », à l'initiative du mouvement "Ni putes ni soumises", réclame la dignité et le respect, dénonce les agressions sur « les filles des quartiers ", de l'insulte au viol et au meurtre; mais, en même temps, leurs porte-parole, étroitement liées à la Maison des Potes, affirment ne vouloir mettre en accusation ni leurs frères, ni la structure familiale, ni aucune religion. Derrière elles, les manifestantes les plus audacieuses, dont certaines battaient déjà le pavé il y a trente ans, osent la formule «le machisme, c'est du fascisme "; du patriarcat, il n'y a nulle évocation.

L'évolution des publications des chercheurs en France dessine la même tendance : à la bibliothèque Marguerite Durand, consacrée à l'histoire des femmes et des féminismes, la vedette " patriarcat » est absente des dossiers constitués par thème et répertorie au catalogue-sujet des ouvrages, bien peu nombreux, antérieurs aux années 1980. Désormais, recourir à ce concept dans le cadre d'une étude sur la France paraît à la fois désuet et démodé. Clémentine Autain dans son invitation au féminisme lui substitue, à la suite de Nicole-Claude Mathieu ${ }^{5}$, le terme "viriarcat » qui, affirme-t-elle, reprend "la logique du mot patriarcat en le réactualisant " ${ }^{6}$. L'usage de celui-ci semble même porteur de désagréments : déplacé, il paraît évoquer moins la structure, qui enchâsse pourtant avec clarté les rapports de sexes, qu'il ne stigmatise de façon péjorative la personne qui l'utilise. Celle-ci risque d'être érigée en une caricature du féminisme du MLF, une nostalgique d'un mythique matriarcat, une partisane d'une guerre des sexes. Ainsi balayé, le patriarcat a déserté les textes; seule reste sa trace sous la forme d'un adjectif, bien doux; il évoque une influence passée en un temps révoqué (du code civil napoléonien marqué du sceau patriarcal aux sociétés méditerranéennes patriarcales, voire au franquisme en appui sur la société espagnole, de tradition catholique et patriarcale...). Alors que jamais la chute du patriarcat n'a été constatée, seuls les anarchistes continuent de s'y référer clairement. La revue No pasaran, solidaires, égalitaires, libertaires, organe du réseau du même nom, met le combat contre le patriarcat à son programme ${ }^{7}$ et y consacre même un numéro spécial au printemps 2003, amenant du reste les intervenants à employer ce mot qui ne leur est plus familier. Hors 
de ce cercle politique restreint, la notion de patriarcat s'est évaporée, du moins sous nos cieux... Son emploi demeure en effet fréquent pour analyser les rapports de sexes en Afrique, ou en Arabie. Spécifique est la position des féministes indiennes : elles désignent clairement au nombre de leurs enjeux contemporains le renversement du patriarcat et les chercheuses fondent sans ambiguïté leur analyse sur l'articulation patriarcat/caste/ capitalisme ${ }^{8}$. Elles rappellent ainsi les positions idéologiques des féministes radicales des années 1970.

Pour celles-ci, à la source de l'inégalité des sexes se trouvait le patriarcat; emprunté au champ anthropologique, il était considéré comme indissociable du système capitaliste par ces militantes issues majoritairement des rangs d'une extrême-gauche qu'elles avaient quittée en constatant que cette mouvance n'était disposée ni à poser la question de l'oppression des femmes, ni à renoncer au pouvoir masculin, celui, entre autres, de ses dirigeants. Leur projet féministe était global : il dépassait largement les thèmes rassembleurs des manifestantes comme la liberté de la contraception et l'avortement ; il prônait de nouveaux rapports de sexes, non par des réformes mais par la destruction du patriarcat et donc nécessairement du capitalisme. L'usage du mot dessinait cependant des frontières entre les féministes socialistes (tendance lutte des classes) qui se gardaient de l'employer et les féministes radicales qui le considéraient comme le concept le plus important dans la lutte des femmes. En intégrant dans leur vocabulaire le terme patriarcat, les historiennes des femmes affichaient ainsi leur volonté de remonter à la source des inégalités sexuées et d'interroger le passé sur cette construction patriarcale afin d'en comprendre les effets sur les rapports de sexes, mais elles ne posaient pas clairement, comme les militantes, l'éventuelle articulation avec le capitalisme. Difficile de saisir la signification de ce silence. En raison de l'étroit lien qui unit, à sa naissance, le Mouvement de libération des femmes et l'histoire des femmes, l'articulation allait-elle de soi ou préférait-on en oublier la portée révolutionnaire pour ne pas politiser davantage, au risque de les décrédibiliser scientifiquement ou de les diviser, les historiennes des femmes? Quoi qu'il en fût, les premiers travaux s'inscrivaient donc dans une logique de dénonciation préconisée par les féministes.

5 Cette approche ne fut sans doute pas étrangère à la tonalité dite victimaire qui domina alors et qui fut si souvent, et aujourd'hui encore, reprochée à l'histoire des femmes. De fait, il n'est pas possible de pointer les dominations, les soumissions, les inégalités, sans identifier clairement les dominants et les dominées. Mais l'approfondissement des recherches montra rapidement que «la dialectique toujours utilisée de la domination et de l'oppression (...) ne sort guère de l'énoncé tautologique dès lors qu'on n'essaie pas d'analyser par quelles médiations spécifiques, dans le temps et dans l'espace cette domination s'exerce $»^{9}$. Loin de $"$ l'autofascination pour le malheur ${ }^{10}$, il s'agissait de rendre compte non d'une structure rigide, mais d'une réalité quotidienne, mouvante et complexe où les rapports de sexes sont sans cesse négociés. Etaient donc proclamées à la fois leur historicité et la non linéarité de la marche vers l'égalité des sexes. Les études repéraient les formes de pouvoir des femmes, leurs actions subversives, leurs espaces de respiration et d'expression, mais aussi l'existence de formes de consentement. Les développements du concept de genre furent sans doute pour beaucoup dans ce déplacement conceptuel. Puisque les rapports de sexes sont une construction socioculturelle - on remarquera l'éviction du terme politique- l'intelligibilité de cette construction par l'histoire du gender en suggère une modification. Histoire du genre et féminisme font cause commune. S'ouvrent ainsi des possibles qui sentent bien plus la réforme que la révolution; ils font, du reste, écho aux luttes des féministes d'antan 
retrouvées par les travaux des années $1980^{11}$ et à celles présentes de la majorité des féministes qui combattent désormais sur le terrain du droit. Aussi les féministes radicales estiment-elles indispensables de défiler le 8 mars 1980 derrière une banderole qui proclame: "Ici on lutte contre le patriarcat", sous-entendant qu'elles sont les seules restées fidèles à ce combat. L'arrivée au pouvoir de la gauche accentue l'évolution et modifie la donne. Le 23 octobre 1982 a lieu une des dernières manifestations derrière une banderole se réclamant du Mouvement ${ }^{12}$. Yvette Roudy entre au gouvernement, porteuse des espoirs de réformes en faveur de l'égalité des sexes. La même année, le Colloque de Toulouse «Femmes, féminisme, recherches" officialise la naissance des "women'studies" à la française. Le gouvernement les baptise en lançant une Action thématique programmée « Recherches sur les femmes et recherches féministes» (1984-1989). Féministes, chercheuses sur les femmes et élites gouvernementales semblent marcher main dans la main. Du patriarcat il n'est donc plus question. L'Histoire des femmes en Occident, parue en 1991-1992, ne lui fait l'honneur que d'un seul titre d'article - "Le Patriarcat fasciste - Mussolini et les Italiennes (1922-1940)»- écrit par Victoria de Grazia. L'historienne regarde la dictature mussolinienne comme "un épisode spécifique du régime patriarcal » ${ }^{13}$, alors même que l'analyse de celui-ci est singulièrement absente des cinq volumes de cet ouvrage collectif.

Dans les sciences humaines et sociales, l'emploi du mot genre participe à cet assagissement de la parole. D'abord employé au singulier, il définit dans un système qui n'est plus interrogé la construction de la différence des sexes, tout en contribuant pourtant à faire disparaître femmes et hommes dans leur singularité. Certes, ce concept a le mérite de mettre l'accent sur la complexité des rapports de sexes, sans séparation, mais peut-on trouver concept plus consensuel : il estompe les aspérités des relations entre les sexes, il en euphémise les rapports sociaux. En même temps que la notion de lutte des classes, celle de lutte des sexes devait-elle aussi disparaître ? L'extension de l'emploi du mot genre affaiblit sa portée: au "sexe du» (du travail, du savoir...) succède le "genre et" (guerre, justice) puis le «genre de » qui permet même de réfléchir sur celui des territoires. Cette extension s'est compliquée dans la seconde moitié des années 1990 par son usage au pluriel : les genres remplacent les sexes... Ce glissement qui commence à faire l'objet de plusieurs débats ${ }^{14}$, obscurcit sans doute la réflexion, aplatit l'analyse que Pierre Bourdieu était venu réactiver dans son livre $L a$ Domination masculine ${ }^{15}$.

7 Pour sortir de ce paravent conceptuel derrière lequel on ne sait plus ce qui se cache, on resexualise l'histoire, sans faire de vague, en parlant du masculin et du féminin, on se réjouit que l'histoire des femmes - non bien sûr de la féminité, à connotation négative débouche sur une histoire de la masculinité, sur la connotation positive de laquelle on ne s'arrête du reste pas ${ }^{16}$. On a soin d'exprimer «les deux genres ", en indiquant le féminin entre parenthèse : les député(e)s; dénoncée comme une marque d'invisibilité, voire d'infériorité, cette convention typographique est aujourd'hui remplacée par un tiret, jugé plus égalitaire : les député-e-s.

Tout ce flou exprime peut-être aussi l'éloignement des années 1970 : les chercheurs en histoire du genre, hommes et femmes, ne sont pas tous issus des rangs féministes ; l'institutionnalisation des recherches, aussi restreinte soit-elle, risque fort d'inviter davantage au consensus, parfois au service d'ambitions carriéristes, qu'au combat idéologique et à l'exigence intellectuelle. Curieuse posture en un temps de 
crise, de chômage, de mondialisation, de montée de l'extrême- droite ; or, plus que les hommes, les femmes sont victimes de ces bouleversements. Mais tout comme le patriarcat, le mot victime est devenu tabou ; ce n'est pas hasard : penser qu'être femme multiplie les risques d'être victime oblige à interroger le système sociétal, à le mettre en cause.

9 La parution du livre d'Elisabeth Badinter, Fausse route ${ }^{17}$, vient tristement confirmer les effets de ces «flous ». Son analyse ne prend pas en compte le fait qu'une société n'est pas une somme d'individus, électrons libres détachés de tout système. Dans le même temps, elle établit une distinction, plus humiliante que subtile, entre les femmes violentées, selon leur groupe social ; seule la situation de celles des quartiers relèverait d'un «féminisme d'urgence». Elle affirme par ailleurs que les violeurs sont tous des malades : leur irresponsabilité dédouane donc la société et coupe court à toute analyse sur le fondement de ces violences, sur leur inscription dans les rapports de sexes. Cette approche de la violence sexuelle pathologisée permet à l'auteure de nier l'existence d'un "être femme» dans la société; celui-ci ne renvoie pas à la biologie, il est le fondement même du lien qui unit depuis toujours les féminismes, malgré les différences de classe et de pays. En se contentant d'accuser la pauvreté et l'injustice comme cause des violences contre les "filles de quartier", en omettant de plus de constater qu'elles subissent la même misère que les hommes mais ne s'en prennent pas à leur intégrité sexuelle, on évacue toute réflexion sur la domination masculine.

Ce vide conceptuel et une semblable confusion entre les mots permettent à Marcela Iacub de prétendre à une sacralisation de la sexualité et de s'en prendre à un soi-disant retour de l'ordre moral venant des féministes ${ }^{18}$. Ces chercheuses pourtant patentées, fortement médiatisées, innocentent le système qui génère les rapports de sexes ; elles voient dès lors dans les démarches de ce qu'elles nomment un nouveau féminisme une "escroquerie, un dévoiement de la lutte des femmes et le retour à la police des mœurs ${ }^{19}$, alors qu'il défend ce qu'il considère être la dignité humaine.

Y.R.

\section{La notion de patriarcat aux États-Unis}

Aux États-Unis, la notion dessine une trajectoire similaire. Le concept de patriarchy tel que nous le comprenons aujourd'hui émerge avec le mouvement féministe des années 1970, pour s'estomper par la suite. Si le sens plus ancien et religieux du terme reste beaucoup plus prégnant en France, des articles des revues scientifiques américaines s'intéressent aussi au patriarcat arménien ou grec. Mais les premiers articles qui utilisent le terme dans un sens plus social le font dans les années 1960, précédant un usage féministe, dans une interprétation plus générale des modes de paternalisme dans les rapports sociaux, qu'il s'agisse de comparaisons entre le Nouveau monde et l'Ancien ou des rapports entre Blancs et Noirs ${ }^{20}$.

13 Le tournant s'opère dans les années 1970, quand le terme patriarchy devient un argument de combat, discuté et bientôt disputé parmi les chercheuses américaines féministes. Une première série d'articles apparaît en 1974, suite à la publication l'année précédente d'un livre polémique de Steven Goldberg intitulé Inevitability of Patriarchy ${ }^{21}$. Comme le résume succinctement Joan Huber, la thèse de Goldberg est que "les hormones forment [shape] les institutions sociales » ... «Goldberg's theory clearly 
shows that biological reductionism is not dead but still twitching ${ }^{22}$. Le livre déclenche une polémique chez les féministes dans la littérature sociologique et anthropo-logique.

Or, c'est l'article de 1976 de l'économiste Heidi Hartmann, «Historical Roots of Occupational Segregation - Capitalism, Patriarchy, and Job Segregation by Sex » qui fera référence ${ }^{23}$. Hartmann souligne des rapports sociaux de sexe au sein du capitalisme, battant en brèche les explications, ou bien néo-classiques ou bien marxistes qui focalisent sur les seuls rapports de capitaux. L'auteure souligne la préexistence du patriarcat sur le capitalisme, qui, d'ailleurs, le mettrait en cause par son inclusion des femmes dans le marché du travail. C'est la nouvelle division/ségrégation $\mathrm{du}$ travail par sexe qui remettrait le patriarcat au sein du système capitaliste. Cette utilisation du terme, liant rapports de sexe, rapports de pouvoir et rapports économiques, connaîtra un essor remarquable. Le nombre d'articles incorporant le terme croît de 12 en 1979 à 25 en 1984, puis à 62 en 1995. Mais le seul nombre est trompeur. Tantôt, les articles soutiennent la thèse de Hartmann, tantôt ils la nuancent ou la critiquent.

15 La critique vient surtout du concept " concurrent ", "gender ", grâce au fameux article de Joan Scott qui est en lui-même un tournant important dans la conceptualisation du champ féministe chez les intellectuels. Dans son article publié en 1986, Joan Scott renverse à son tour les schémas en soulignant que le patriarcat ne peut pas être compris comme une donnée universelle, a-historique ${ }^{24}$. En introduisant le concept de "genre » sexuel, en mettant l'accent sur la construction sociale des rapports entre hommes et femmes, Joan Scott lance un terme qui va avoir sa propre vie, balayant d'une certaine manière la réflexion autour du patriarcat et de la domination masculine qu'il implique.

La réflexion autour de la notion de patriarcat, en relation avec d'autres formes d'exploitation, n'est pas pour autant terminée. Si elle semble quelque peu désuète (trop militante, comme le suggère Yannick Ripa) et donc moins utilisée aux États-Unis ou en France, des militantes et chercheurs en Chine, en Inde, ou ailleurs, semblent l'avoir repris à bon escient.

17 N.L.G.

\section{Féminisme, politique et pouvoir}

18 Au départ - expression qui renvoie à ce qu'on appelait «l'année zéro » du féminisme tant il était vrai que nous avions le sentiment de ne pas avoir d'histoire - au départ, «nous»- ce «nous» était surtout composé de femmes assez jeunes, de milieu bourgeois, majoritairement blanches, "éduquées » et occidentales -, au départ, "nous» étions libertaires, nous nous méfiions des partis politiques, nous ne voulions pas réformer la société, mais nous voulions changer le monde. On parlait de révolution. L'ennemi, c'était le patriarcat, c'était aussi (souvent) nos mères, jugées complices. Il fallait s'inventer. Ce refus de pactiser avec le pouvoir en place ou d'adopter ses pratiques se faisait pour certaines d'entre nous au nom de l'idée de la révolution permanente et indépendante des fins qu'elle poursuit. Cette idée s'est vite radicalisée, légitimant les extrêmes, profitant d'une béance (ou peut-être du désarroi) du pouvoir en place. 

se conjuguent avec les moments forts de lutte sociale et politique. Le mouvement féministe en 1970 était en phase avec les grands mouvements de libération nationale dans le monde, avec les grandes luttes ouvrières. Cela signifiait que nous n'avions pas à nous poser la question d'un féminisme international ou transnational ni à étudier comment nous nous situions par rapport aux féminismes du "Tiers-Monde», dont nous ne savions pas grand chose : les luttes de libération et la nébuleuse encore vivace de l'internationale marxiste suffisaient à nous insuffler force et courage.

Une citation du Torchon brûle illustre mon propos. Un article intitulé "Les femmes" reprend une formulation de l'activiste noir Huey P. Newton: «le pouvoir, c'est la capacité à définir un phénomène et à le transformer en action selon nos désirs. » Il s'agissait de s'appuyer sur cette démarche pour combattre tout ce que les femmes avaient intériorisé comme domination. L'analyse des rapports de force ne se traduisait pas en stratégies spécifiques : les « coups " tactiques, si possible médiatisés, formaient l'essentiel de l'action et pour le reste, nous étions portées par le souffle utopique des débuts. La prise de conscience de nos forces suffisait à décupler les énergies. Sisterhood is powerful, ce slogan venait des États-Unis, on l'avait adopté, on y croyait. Dans un universalisme de bon aloi, la sororité gommait les différences et l'enthousiasme provoquait comme une grande vague d'espoir.

21

Nous étions certes en phase mais singulières, ayant compris la nécessité historique d'une séparation d'avec les autres luttes afin d'obtenir certaines avancées concernant d'abord et surtout les femmes (en particulier les acquis des lois contre le viol et pour l'avortement), afin aussi de tenter d'élaborer une théorie et une pratique qui dépendaient, pour une large part, des formations que certaines avaient déjà acquises dans les organisations politiques de gauche et les syndicats ainsi que dans les « lieux de savoir »: l'université et la culture psychanalytique. On ne parlait pas encore de domination masculine mais du pouvoir des hommes, de la "politique du mâle». Si «la » politique était bannie du vocabulaire dans le mouvement des femmes, en pratique des avocates et des militantes du MLAC menaient des pourparlers avec « le pouvoir » en place et « le » politique prenait parmi nous une place grandissante.

Il s'agissait de développer un contre-pouvoir. Des femmes? Cela a été dit, mais peu théorisé et vite pulvérisé par une atomisation des points de vue. Souvenons-nous du slogan " hétéro-collabo", slogan qui recouvrait un début de réflexion politique sur le consentement volontaire à la servitude mais qui eut comme effet de provoquer des schismes parmi les féministes. Il est temps de dire ici la dette, souvent non reconnue, $\mathrm{du}$ mouvement de libération des femmes envers les lesbiennes féministes de la première heure, celles qui - étant de par leur condition doublement exclues du pouvoir - ont su donner une impulsion radicale à la pensée féministe. Elles ont posé les jalons d'une théorisation du patriarcat et nous ont donné une grande figure poétique, peu reconnue en France, Monique Wittig.

Reprenons à partir de ce "départ »: un mouvement de libération des femmes, sans structure associative, sans groupes de pression réformistes, qui se voulait sans têtes, sans « cheffes » (au moins dans les discours), tant il était souhaité qu'il soit multiforme et libre. D'où le refus des noms propres : il n'y avait que des Françoises, des Moniques, des Catherines et des Michelles, femmes qui en devenaient anonymes, cachées derrière les prénoms de notre génération. Refusant les patronymes, « elles » donnèrent du fil à retordre aux historiennes qui, plus tard, voulurent faire l'histoire de ce féminisme-là.

Clio. Femmes, Genre, Histoire, 21 | 2005 
Notons l'ironie de la chose. On voulait tirer les femmes de l'anonymat dans lequel elles avaient été plongées historiquement et on le redoublait, ce qui semble aujourd'hui avoir été un piège. Comment avoir du pouvoir si on n'a pas de noms? Et certaines, qui se disaient féministes, ont su manipuler « les autres », les «sans-noms ». L'inscription du sigle MLF par le groupe «Psych et Po» comme marque déposée au Tribunal de Commerce en est un exemple.

24 Au départ, nous, c'était aussi les femmes/féministes qui - jeunes et moins jeunes avaient déjà une formation universitaire ou qui étaient en train d'en acquérir une ; nous voulions - dans l'université ou dans un hors champ universitaire - faire travailler notre savoir, l'élaborer, le détourner et le faire fructifier en prenant comme objet d'étude les femmes. L'examen des rapports entre l'être (au féminin) écrit et/ou représenté et l'être écrivant (les femmes écrivant dont nous étions) constituait un enjeu, une gageure et permettait à certaines d'entre nous un investissement à la fois intellectuel et existentiel. Vouloir faire entrer dans nos disciplines ce champ nouveau répondait aussi à un désir de légitimation de notre part, désir hautement politique. On était encore loin de la professionnalisation des études sur les femmes, les luttes internes dans les universités et les centres de recherche ne faisaient que commencer, la rupture entre engagement militant et retrait dans les archives n'était pas effectuée même si elle était vite pressentie. Pendant les années 1970, on pouvait encore mener plusieurs actions de front. La recherche féministe n'était pas encore coupée de la pratique militante. Le slogan «l'individuel est politique » menait aux études sur le travail domestique et à l'articulation politique entre le privé et le public ${ }^{25}$.

251981 marque un changement. D'abord, avec l'élection de François Mitterrand, le mouvement cesse, en grande partie, d'être dans l'opposition. Il est dorénavant possible d'obtenir des subventions, de former des groupes associatifs, d'entrer dans une logique réformiste. La nouvelle réalité freine la radicalité du mouvement, puis la fait disparaître. Il n'y a plus guère de grandes manifestations de femmes, plus de femmes dans la rue. Le reflux du féminisme des années 1970 est enclenché. Le féminisme avance masqué dans les instances du pouvoir.

26 En même temps, dans ces années-là, les grandes luttes de libération nationale se sont effondrées ou ont été récupérées, institutionnalisées. Le colonialisme, façon dixneuvième siècle prend de nouvelles formes avec les débuts d'une nouvelle mondialisation. L'ère post-coloniale est peu analysée par les féministes qui, le plus souvent, n'en saisissent pas les enjeux pour les femmes. L'Europe se constitue mais la plupart des féministes ne s'organisent pas en fonction de cette nouvelle donne politique. D'ailleurs, en général, les relations internationales ne suscitent guère l'intérêt de l'ensemble des féministes; peu d'entre elles s'engagent dans la recherche en sciences politiques, et les actions ponctuelles de solidarité dans les moments de crises à travers le monde ne débouchent pas sur une action concertée.

Néanmoins, c'est dans les années 1980 que se développe une pensée solide dans certains lieux de recherche, surtout en sciences sociales et, dans une moindre mesure, en lettres. Le refus de se cantonner dans la victimisation et l'invariant d'une oppression, pour étudier les avancées et les reculs, pour ouvrir des espaces de réflexion sur les rapports sociaux de sexe - $\mathrm{y}$ compris ceux où le bonheur et la séduction jouaient un rôle -, sont autant de points forts de la pensée féministe et constituent des acquis durables pour l'outillage de la réflexion sociale et politique. Citons ici un exemple : en 1986, un article des Annales, intitulé "Culture et pouvoir des femmes: essai 
d'historiographie " porte une attention soutenue aux mots " pouvoir » et " politique " et retrace le cheminement de la pensée féministe (occidentale) sur la culture des femmes. ${ }^{26}$ Étudier comment les femmes pouvaient avoir « du pouvoir », c'était ouvrir de nouvelles grilles de lecture du passé et du présent; réfléchir à un rapport politique à partir des conditions faites aux femmes, c'était penser comment elles pouvaient (ou pas) avoir «le pouvoir ». L'enjeu politique consistait à réévaluer à l'aune du politique différents événements où les femmes participent à l'histoire. Malgré le travail accompli, le "repli identitaire " de ce qui était devenu un petit "sous groupe », celui des féministes dans le champ des sciences sociales, est douloureux, malgré les succès individuels ou arrachés par plusieurs groupes associatifs. Les féministes " historiques » se sentent souvent coupées des femmes plus jeunes, des femmes «en général ». Cette coupure - sans doute plus apparente que réelle - est largement alimentée par les médias. L'anti-féminisme, de retour en force, se manifeste dans la publicité et les écrits. La femme "jetable», comme l'homme "jetable», est une réalité commerciale qui semble nouvelle parce qu'elle a pris une nouvelle forme. L'effondrement généralisé des équilibres mondiaux ainsi que la mondialisation capitaliste du commerce se précisent tout au long des années 1990 et ce n'est pas un hasard si on peut déclarer que les « décennies libérales (1980-2000) se sont révélées particulièrement brutales à l'égard des femmes ${ }^{27}$.

C'est dans ce climat que la lutte réformiste pour la parité (l'obligation de présenter un nombre égal d'hommes et de femmes dans la plupart des listes électorales à tous les niveaux de la représentation politique) engage certaines féministes ainsi que les grandes associations féminines françaises (1992-2000). L'impulsion pour la parité politique est donnée par l'Europe, la France étant désignée comme « la lanterne rouge » de l'Union en ce qui concernait les femmes élues démocratiquement. Le grand débat qui s'enclenche alors redonne au féminisme en France une force et une légitimité aux yeux de l'opinion publique qu'il avait perdues depuis une décennie. Il provoque en même temps un désaccord entre féministes et un nouveau "mot phare " qui était absent des débats du MLF apparait. Il s'agit du mot «République », des idéaux et des réalités que ce concept politique recouvre. L'idéal de la République, cher à beaucoup de féministes mais interprété différemment selon les convictions politiques, fait prendre parti pour ou contre la parité. La crainte d'un multiculturalisme menaçant la laïcité et la neutralité d'une République qui se voudrait sans sexe, sans religion et sans appartenance ethnique, suscite des propos suggérant que des "quotas» de femmes ouvriraient la boîte de Pandore du communautarisme en France.

S'il est entendu que la parité dans ses effets ne donne " du pouvoir » qu'à une petite minorité de femmes de classe moyenne, la problématique, par contre, repose la question de l'universel et aurait pu permettre de penser le féminisme en dehors d'une spécificité franco-française et d'un repli sur des positions peut-être trop étroitement nationalistes. Il faut constater que c'est le contraire qui se produit aujourd'hui dans " l'affaire du foulard » qui divise de nouveau les féministes, comme la classe politique et la société civile. Permettre ou non à certaines jeunes filles françaises et musulmanes portant le foulard de suivre une scolarité normale dans l'éducation secondaire, laïque et républicaine est re-devenu une affaire d'État depuis les événements du 11 septembre 2001 et la peur généralisée d'un terrorisme musulman que ceux-ci ont suscitée. Les féministes se rangent, comme bien des partis et des mouvements d'idées, ici encore, soit contre l'accès à l'école de ces jeunes filles, au nom des valeurs de la République et de la subordination des femmes incarnée par « le voile » selon la plupart des Françaises, 
soit pour une vision élargie d'une République qui permettrait un droit à la différence et qui aménagerait l'accueil des pluralismes à l'intérieur de ses institutions les plus chères $^{28}$. La loi excluant les jeunes filles portant foulard est appliquée depuis septembre 2004. Dans ce débat, fortement médiatisé et instrumentalisé par le pouvoir politique en place, peu de temps de parole est donné aux Françaises musulmanes pratiquantes et féministes et le dialogue qui pourrait s'installer ne semble pas avoir lieu ${ }^{29}$. L'ignorance de l'islam, l'amalgame constamment pratiqué entre toutes les utilisations du voile et la présupposition que les femmes portant le foulard ne possède aucune mesure de librearbitre renvoient aux « autres » une image d'un féminisme des dominant(e)s, frileux et condescendant.

En général, peu d'attention est accordée aux enjeux internationaux que les luttes féministes internes reflètent sans en tenir compte. Penser globalement, agir localement ${ }^{30}$ est un slogan qui mériterait une réflexion féministe approfondie. La gauche française dans laquelle se situe une grande partie du féminisme parvient mal à relativiser un eurocentrisme qui la fait se prendre pour le centre du monde et des valeurs. On constate néanmoins qu'une nouvelle mobilisation des femmes se fait, cette fois dans la mixité, non seulement en France où des luttes que les médias nous disaient terminées sont d'actualité mais aussi au sein des grandes luttes alter-mondialistes. Parmi les ateliers du Forum social mondial qui se tenait à Porto Alegre, en 2003, un atelier intitulé «le féminisme et la nouvelle génération politique» a attiré de jeunes féministes. Le débat a tourné autour du féminisme, de l'espace des femmes dans les groupes et coalitions de mouvements qui s'opposent à la mondialisation néo-libérale et des dynamiques entre générations au sein du mouvement féministe. À l'occasion de cette activité a commencé à prendre forme un réseau international pour bâtir et renforcer le mouvement féministe parmi «les nouvelles générations ». ${ }^{31}$ Mais les «jeunes féministes » citées plus haut, si elles sont souvent européennes, ne sont que rarement françaises. Néanmoins, les fortes mobilisations de femmes autour la Marche des Femmes et l'Assemblée Européenne pour les Droits des Femmes qui a eu lieu en novembre 2003 à Bobigny et a réuni plus de 2000 personnes représentant 160 associations et organisations de 37 pays sont autant de signes de renouveau.

31 Terminons par un constat. Pour le mouvement féministe et les études féministes, il serait bon d'être à la fois "chez nous » et en phase avec les mouvements de lutte qui s'engagent dans le monde.

\section{BIBLIOGRAPHIE}

Mouvement social, 2002, janvier-mars, n 198, « Féminin et masculin » (dir. Anne-Marie Sohn).

AUTAIN Clémentine, 2001, Alter égaux, Invitation au féminisme, Paris, Robert Laffont.

BADINTER Elisabeth, 2003, Fausse route, Paris, Odile Jacob.

BOURDIEU Pierre, 1998, La Domination masculine, Paris, Seuil. 
BOUZAR Dounia et KADA Saïda, 2003, L'une voilée, l'autre pas, Paris, Albin Michel.

DAUPHIN Cécile et al., 1986, «Culture et pouvoir des femmes : essai historiographique », Annales ESC, 41, p. 271-293.

DELPHY Christine, 1996, L'ennemi principal, 1-Économie politique du patriarcat, Paris, Syllepse.

--, 2001, L'ennemi principal, 2-Penser le genre, Paris, Syllepse.

DEUDON Catherine, 2003, Un Mouvement à soi. Images du mouvement des femmes, 1970-2001, Paris, Syllepse.

DUBY Georges et PERROT Michelle (dir.), 1991-1992, Histoire des femmes, Paris, Plon, 5 vol.

GOLDBERG Steven, 1973, Inevitability of Patriarchy, New York, William Morrow \& co.

HAASE-DUBOSC Danielle et VIENNOT Eliane, 1991, Femmes et Pouvoirs sous l'Ancien Régime, Paris/ Marseille, Editions Rivages.

HAASE-DUBOSC Danielle et al. (éd.), 2002, Enjeux contemporains du féminisme indien, Paris, Edition de la Maison des sciences de l'homme.

HÉRITIER Françoise, 1996, Masculin/féminin. La pensée de la différence, Paris, Odile Jacob.

--, 2002, Masculin/féminin. Dissoudre la hiérarchie, Paris, Odile Jacob.

HIRATA Helena et al., 2002, Dictionnaire critique du féminisme, Paris, PUF.

IACUB Marcela, 2002, Qu'avez-vous fait de la libération sexuelle?, Paris, Flammarion.

KLEJMAN Laurence et ROCHEFORT Florence, 1989, L'Égalité en marche. Le féminisme sous la Troisième République, Presses FNSP/des femmes.

LAUFER Jacqueline et al., 2003, Le Travail du genre, les sciences sociales du travail à l'épreuve des différences de sexe, Paris, La Découverte/Mage.

MATHIEU Nicole-Claude, 1985, L'arraisonnement des femmes, Paris, EHESS.

SCOTT Joan Wallach, 1986, « Gender : a useful category of historical analysis », American Historical Review, vol. 91, 5 (traduit in Les Cahiers du GRIF, n 37-38, « Le genre de l'histoire », 1988).

\section{NOTES}

1. Delphy 1996 et 2001.

2. Héritier 1996 et 2002.

3. Christine Delphy rappelle qu'avant le féminisme, seul le qualificatif était employé, dans un sens positif et relié comme chez Victor Hugo à la famille (des vertus patriarcales), car « il est de la nature du patriarcat - comme de tout système d'oppression - de se nier en tant que tel », in Delphy $1996: 224$.

4. Pour exemple : «Tant qu'il y aura des femmes à baiser/ Les bas Dim feront des affaires/ Tant qu'il y aura des hommes pour juger/Le patriarcat sera rassuré » (1973) ; « Avec leurs perceus's électriques/ Et leurs bombes très atomiques/Avec leur tiercé du dimanche/ Et leurs tondeuses à gazon/ Ils brament à la castration/ C'est le patriarcat qui flanche », Chanson du film vidéo Maso et Miso vont en bateau (1976) ; «A bas l'ordre bourgeois/Et l'ordre patriarcal/ A bas l'ordre hétéro et l'ordre capitalo », Tendance désordre des gouines rouges, 1971.

5. Mathieu 1985.

6. Autain $2001: 40$. 
7. Le réseau No pasaran se définit comme « issu du mouvement antifasciste radical. Son analyse de la montée de l'extrême droite et des idéologies xénophobes, sécuritaires et autoritaires, et son expérience de lutte antifasciste l'ont amené à élargir son champ d'intervention. Le Réseau No pasaran combat toutes les formes de domination: capitalisme, racisme, patriarcat, aliénation, apartheid social, répression. » (en gras dans le texte), la revue est publiée par la maison d'édition du Réseau, Reflex.

8. Haase-Dubosc et al. 2002.

9. Dauphin et al. $1986: 273$.

10. Ibidem.

11. Particulièrement ceux de Klejman et Rochefort 1989.

12. Deudon 2003.

13. Duby et Perrot 1992, vol. $5: 115$.

14. Voir Laufer et al. 2003. Et plus particulièrement, l'article de Françoise Thébaud, « Histoire des femmes, histoire du genre et sexe du chercheur », p. 70-87.

15. Bourdieu 1998.

16. Tel est le thème du numéro du Mouvement social de janvier-mars 2002.

17. Badinter 2003.

18. Iacub 2002.

19. Interview croisée d'Elisabeth Badinter et Marcela Iacub intitulée « Ordre moral et sexualité. Le nouveau féminisme, 'cette escroquerie' », in Le Nouvel Observateur, 14 mai 2003, p. 92.

20. En recherchant des articles utilisant le mot «patriarchy » dans le titre dans le Social Science Citation Index à partir de 1956, les trois premiers articles sont : P. Conner, « Patriarchy : Old World and New", American Quarterly, vol.17, 1, p. 48-62; A.B. Lewis, «Peasant Family Operation Between Patriarchy and Partnership», Rural Sociology (compte rendu), vol. 31, 2, p. 233-34; B. Hayne, "Yankee in Patriarchy - Thorpe, TB Reply to Uncle Tom's Cabin », American Quarterly, vol. 20, 2, p. 180-195.

21. Goldberg 1973.

22. "La théorie de Golderg montre clairement que la réduction au biologique n'est pas morte mais bouge encore. » Joan Huber, compte rendu de l'ouvrage de Goldberg in American Journal of Sociology, 1974, vol. 80, 2, p. 567-569.

23. Signs, vol. 1, 3, p. 137-169. Il s'agit donc bien de 1976 et non pas de 1981 (quand l'article reparaît dans un ouvrage collectif), date cité dans Hirata $2000: 144$.

24. Scott 1986.

25. Un exemple parmi d'autres : le titre «Regards intimes et politiques » du film tourné un an après la disparition du journal féministe, Histoires d'elles en 1981, indique la jonction : nous avons pris conscience d'une force collective, nous étions sorties d'un individualisme stérile.

26. Dauphin et al. 1986. Il faut renvoyer à l'ensemble de cet article pour son analyse des travaux menés sur les problématiques du féminin/masculin et les cultures des femmes. Egalement Duby et Perrot 1991 ; Haase-Dubosc et Viennot 1991.

27. Voir le carnet 68 Manière de voir du Monde Diplomatique, Avril Mai 2003, « Femmes Rebelles ».

28. Voir l'appel « Laïcardes, puisque féministes », lancé dans le journal Le Monde, juin, 2003, dans lequel Anne Vigerie et Anne Zelensky instituent un rapport de stricte équivalence entre le féminisme, la laïcité et l'exclusion des élèves « voilées » ainsi que la réponse de Sylvie Tissot, « Un féminisme à visage inhumain », pris dans le courrier électronique de La marche des femmes, 20 juin 2003, dans lequel elle annonce que «nous sommes contre l'exclusion parce que laïques et féministes ». Cette dernière met en garde contre une philosophie politique terrifiante "qui décrète une partie de la population 'aliénée' et inapte à toute pensée et à toute prise de parole, pour ensuite la 'forcer à être libre'. "

29. Pour des points de vue de musulmanes françaises, voir Bouzar et Kada 2003.

30. Slogan lancé par le mouvement ATTAC fondé en 1998. 
31. Elsa Beaulieu, Marche mondiale des femmes - Québec, Julia Di Giovanni, Marche mondiale des femmes - Brésil. Marchfem@mail.ras.eu.org et http://www.ffq.qc.ca/marche2000.

\section{RÉSUMÉS}

Militante et réflexive, l'histoire des femmes a aussi besoin d'exprimer ses doutes et ses inconforts. Rattrapée par l'actualité, la violence et nombre d'événements cruels et tragiques, par des incertitudes majeures vécues par l'ensemble du monde intellectuel, elle pose aujourd'hui en cet article collectif des interrogations et des inquiétudes. Ce travail à plusieurs (les participantes du Groupe d'histoire des femmes du $\mathrm{CRH}$ ) cherche à mettre à plat ce qui actuellement se dérobe à notre connaissance, en partant de notions-clés souvent utilisées, comme consentement, sexualité, écriture, patriarcat, etc. Chacune de ces notions est discutée à la lumière des brouillages actuels, des temporalités nouvelles, de l'avènement triomphant du corps marchandise, de la pauvreté grandissante, etc.

Women's history, a militant and self-reflexive endeavor, needs to express its doubts and its difficulties. The contemporary world, with its violence, its cruel and tragic events, raises questions for all intellectuals and academics, affected by the uncertainties of the present, and all the more so for women historians and historians of women and gender. This collective article (written by the participants of the Women's History Group of the Centre de Recherches Historiques - CNRS/EHESS) addresses these issues by returning to key concepts that have been utilized over the last few decades - such as consent, sexuality, patriarchy, etc. Each term is analyzed in the light of current debates and new temporalities, from recent discussions about the body (and its commercialization) to growing inequalities, etc.

\section{INDEX}

Mots-clés : classes sociales, histoire des femmes, consentement, écriture, féminisme, paroles, patriarcat, politique, sexualité 